\title{
Higgs mechanism and cosmological constant in $N=1$ supergravity with inflaton in a vector multiplet
}

\author{
Yermek Aldabergenov ${ }^{1, a}$, Sergei V. Ketov ${ }^{1,2,3, b}$ \\ ${ }^{1}$ Department of Physics, Tokyo Metropolitan University, Minami-ohsawa 1-1, Hachioji-shi, Tokyo 192-0397, Japan \\ ${ }^{2}$ Kavli Institute for the Physics and Mathematics of the Universe (IPMU), The University of Tokyo, Chiba 277-8568, Japan \\ ${ }^{3}$ Institute of Physics and Technology, Tomsk Polytechnic University, 30 Lenin Ave., Tomsk 634050, Russian Federation
}

Received: 2 March 2017 / Accepted: 3 April 2017 / Published online: 12 April 2017

(C) The Author(s) 2017. This article is an open access publication

\begin{abstract}
The $N=1$ supergravity models of cosmological inflation with an inflaton belonging to a massive vector multiplet and spontaneous SUSY breaking after inflation are reformulated as the supersymmetric $U(1)$ gauge theories of a massless vector superfield interacting with the Higgs and Polonyi chiral superfields, all coupled to supergravity. The $U(1)$ gauge sector is identified with the $U(1)$ gauge fields of the super-GUT coupled to supergravity, whose gauge group has a $U(1)$ factor. A positive cosmological constant (dark energy) is included. The scalar potential is calculated, and its de Sitter vacuum solution is found to be stable.
\end{abstract}

\section{Introduction}

PLANCK observations [1-3] of the Cosmic Microwave Background $(\mathrm{CMB})$ radiation favor chaotic slow-roll inflation in its single-field realization, i.e. the large-field inflation driven by a single scalar called inflaton with an approximately flat scalar potential.

Embedding inflationary models into $N=1$ fourdimensional supergravity is needed to connect them to particle physics theory beyond the Standard Model of elementary particles and to quantum gravity. Most of the literature about inflation in supergravity is based on an assumption that the inflaton belongs to a chiral (scalar) multiplet - see e.g., the reviews [4,5]. However, the inflaton can also be assigned to a massive $N=1$ vector multiplet. It has some theoretical advantages because there is only one real scalar in an $N=1$ massive vector multiplet. The $\eta$-problem does not arise because the scalar potential of a vector multiplet in supergravity is of the $D$-type instead of the $F$-type. The minimal inflationary models with the inflaton belonging to a mas-

\footnotetext{
a e-mail: aldabergenov-yermek@ed.tmu.ac.jp

be-mail: ketov@tmu.ac.jp
}

sive vector multiplet were constructed in Ref. [6] by exploiting the non-minimal self-coupling of a vector multiplet to supergravity [7]. The supergravity inflationary models [6] have the single-field scalar potential given by an arbitrary real function squared. Those scalar potentials are always bounded from below and allow any desired values of the CMB observables $n_{s}$ and $r$. However, the minima of the scalar potentials of [6] have a vanishing cosmological constant and a vanishing Vacuum Expectation Value (VEV) of the auxiliary field $D$, so that they allow only Minkowski vacua where supersymmetry is restored after inflation.

A simple extension of the inflationary models [6] was proposed in Ref. [8] by adding a Polonyi (chiral) multiplet [9] with a linear superpotential. The inflationary models [8] also accommodate arbitrary values of $n_{s}$ and $r$, and have a Minkowski vacuum after inflation, but with spontaneously broken supersymmetry (SUSY). In this paper we further extend the models of Ref. [8] by allowing them to have a positive cosmological constant, i.e. a de Sitter vacuum after inflation.

Yet another motivation comes from an exposition of the super-Higgs effect in supergravity by presenting the new $U(1)$ gauge-invariant form of the class of inflationary models under investigation. This paves the way toward embedding our models into the superymmetric Grand Unification Theories (sGUT) coupled to supergravity, when they have a spontaneously broken $U(1)$ factor in the sGUT gauge group. The physical scale of cosmological inflation can be identified with the Hubble (curvature) scale $H \approx 10^{14} \mathrm{GeV}$ or the inflaton mass $m_{\mathrm{inf}} \approx 10^{13} \mathrm{GeV}$. The inflationary scale is thus less (though not much less!) than the sGUT scale of $10^{16} \mathrm{GeV}$. The simple sGUT groups $S U(5), S O(10)$ and $E_{6}$ are well motivated in the Calabi-Yau compactified heterotic strings, however, they usually come with at least one extra "undesired" $U(1)$ factor in the gauge group. The well-known examples include the gauge symmetry break- 
ing $E_{6} \rightarrow S O(10) \times U(1), S O(10) \rightarrow S U(5) \times U(1)$, and the "flipped" $S U(5) \times U_{X}(1)$ sGUT originating from heterotic strings. Exploiting the Higgs mechanism in supergravity allows us to propose an identification of the $U(1)$ gauge vector multiplet of those sGUT models with the inflaton vector multiplet we consider, thus unifying inflation with those sGUT in supergravity. Besides the sGUT gauge unification, related proton decay and baryon number violation, having the $U(1)$ factor in the sGUT gauge group allows one to get rid of monopoles, because the gauge group is not semisimple [10]. Having a positive cosmological constant takes into account dark energy too.

Our paper is organized as follows. In Sect. 2 we briefly review the supergravity models [8]. In Sect. 3 we present their $U(1)$ gauge-invariant formulation and the Higgs mechanism. A positive cosmological constant is added in Sect. 4. The scalar potential and it stability are studied in Sect. 5. Our conclusion is given by Sect. 6 .

\section{Scalar potential and SUSY breaking with a massive vector multiplet in the absence of a cosmological constant}

The inflationary models of Ref. [8] are defined in curved superspace of $N=1$ supergravity [11] by the Lagrangian $\left(M_{\mathrm{Pl}}=1\right)^{1}$

$$
\begin{aligned}
\mathcal{L}= & \int \mathrm{d}^{2} \theta 2 \mathcal{E}\left\{\frac{3}{8}(\overline{\mathcal{D} D}-8 \mathcal{R}) e^{-\frac{1}{3}(K+2 J)}+\frac{1}{4} W^{\alpha} W_{\alpha}+\mathcal{W}\right\} \\
& + \text { h.c. },
\end{aligned}
$$

in terms of chiral superfields $\Phi_{i}$, representing ordinary (other than inflaton) matter, with a Kähler potential $K=K\left(\Phi_{i}, \bar{\Phi}_{i}\right)$ and a chiral superpotential $\mathcal{W}=\mathcal{W}\left(\Phi_{i}\right)$, and interacting with the vector (inflaton) superfield $V$ described by a real function $J=J(V)$ and having the superfield strength $W_{\alpha} \equiv-\frac{1}{4}(\overline{\mathcal{D} D}-8 \mathcal{R}) \mathcal{D}_{\alpha} V$. We have also introduced the chiral density superfield $2 \mathcal{E}$ and the chiral scalar curvature superfield $\mathcal{R}$ [11].

After eliminating the auxiliary fields and changing the initial (Jordan) frame to an Einstein frame, the bosonic part of the Lagrangian (1) reads [8]

$$
\begin{aligned}
e^{-1} \mathcal{L}= & -\frac{1}{2} R-K_{i j *} \partial_{m} A_{i} \partial^{m} \bar{A}_{j}-\frac{1}{4} F_{m n} F^{m n} \\
& -\frac{1}{2} J^{\prime \prime} \partial_{m} C \partial^{m} C-\frac{1}{2} J^{\prime \prime} B_{m} B^{m}-\mathcal{V},
\end{aligned}
$$

and it has the scalar potential

\footnotetext{
$\overline{1}$ Our notation and conventions coincide with the standard ones in Ref. [11], including the spacetime signature $(-,+,+,+)$. The $N=1$ superconformal calculus [6,7] after the superconformal gauge fixing is equivalent to the curved superspace description of $N=1$ Poincare supergravity.
}

$$
\begin{aligned}
\mathcal{V}= & \frac{1}{2} J^{\prime 2}+e^{K+2 J}\left[K_{i j^{*}}^{-1}\left(\mathcal{W}_{i}+K_{i} \mathcal{W}\right)\left(\overline{\mathcal{W}}_{j}+K_{j^{*}} \overline{\mathcal{W}}\right)\right. \\
& \left.-\left(3-2 \frac{J^{\prime 2}}{J^{\prime \prime}}\right) \mathcal{W} \overline{\mathcal{W}}\right]
\end{aligned}
$$

where we have introduced the vierbein determinant $e \equiv$ $\operatorname{det} e_{m}^{a}$, the spacetime scalar curvature $R$, the complex scalars $A_{i}$ as physical components of $\Phi_{i}$; the real scalar $C$ and the real vector $B_{m}$, with the corresponding field strength $F_{m n}=\mathcal{D}_{m} B_{n}-\mathcal{D}_{n} B_{m}$, as physical components of $V$. The functions $K, J$ and $\mathcal{W}$ now represent the lowest components $\left(A_{i}\right.$ and $C$ ) of the corresponding superfields. As regards their derivatives, we use the notation $K_{i} \equiv \frac{\partial K}{\partial A_{i}}, K_{i^{*}} \equiv \frac{\partial K}{\partial \bar{A}_{i}}$, $K_{i j^{*}} \equiv \frac{\partial^{2} K}{\partial A_{i} \partial \bar{A}_{j}}, J^{\prime} \equiv \frac{\partial J}{\partial C}, \mathcal{W}_{i} \equiv \frac{\partial \mathcal{W}}{\partial A_{i}}, \overline{\mathcal{W}}_{i} \equiv \frac{\partial \mathcal{W}}{\partial \bar{A}_{i}}$. As is clear from Eq. (2), the absence of ghosts requires $J^{\prime \prime}(C)>0$, where the primes denote differentiations with respect to the given argument. ${ }^{2}$

For our purposes here, we restrict ourselves to a single chiral superfield $\Phi$ whose Kähler potential and the superpotential are those of the Polonyi model [9]:

$K=\Phi \bar{\Phi}, \quad \mathcal{W}=\mu(\Phi+\beta)$,

with the parameters $\mu$ and $\beta$. The choice (4) is quite natural (and unique) for a nilpotent (Volkov-Akulov) chiral superfield $\Phi$ obeying the constraint $\Phi^{2}=0$, though we do not employ the nilpotency condition here, in order to avoid its possible clash with unitarity at high energies.

A substitution of Eq. (4) into the Lagrangian (2) yields

$$
\begin{aligned}
e^{-1} \mathcal{L}= & -\frac{1}{2} R-\partial_{m} A \partial^{m} \bar{A}-\frac{1}{4} F_{m n} F^{m n}-\frac{1}{2} J^{\prime \prime} \partial_{m} C \partial^{m} C \\
& -\frac{1}{2} J^{\prime \prime} B_{m} B^{m}-\frac{1}{2} J^{\prime 2}-\mu^{2} e^{A \bar{A}+2 J}\left[|1+A \beta+A \bar{A}|^{2}\right. \\
& \left.-\left(3-2 \frac{J^{\prime 2}}{J^{\prime \prime}}\right)|A+\beta|^{2}\right],
\end{aligned}
$$

where the complex scalar $A$ is the lowest component of the Polonyi chiral superfield $\Phi$.

The Minkowski vacuum conditions,

$$
\begin{aligned}
V=\frac{1}{2} J^{\prime 2}+\mu^{2} e^{A \bar{A}+2 J}\left[|1+A \beta+A \bar{A}|^{2}\right. \\
\left.-\left(3-2 \frac{J^{\prime 2}}{J^{\prime \prime}}\right)|A+\beta|^{2}\right]=0 \\
\partial_{\bar{A}} V=\mu^{2} e^{A \bar{A}+2 J}[A(1+\bar{A} \beta+A \bar{A})+(A+\beta) \\
\quad \times(1+A \beta+A \bar{A})-\left(3-2 \frac{J^{\prime 2}}{J^{\prime \prime}}\right)(A+\beta) \\
\left.+A|1+A \beta+A \bar{A}|^{2}-\left(3-2 \frac{J^{\prime 2}}{J^{\prime \prime}}\right) A|A+\beta|^{2}\right]=0,
\end{aligned}
$$

\footnotetext{
${ }^{2}$ Our $J$-function differs by the sign from that in Refs. [6,7].
} 


$$
\begin{aligned}
\partial_{C} V= & J^{\prime}\left\{J^{\prime \prime}+2 \mu^{2} e^{A \bar{A}+2 J}\left[|1+A \beta+A \bar{A}|^{2}\right.\right. \\
& \left.\left.-\left(1-2 \frac{J^{\prime 2}}{J^{\prime \prime}}+\frac{J^{\prime} J^{\prime \prime \prime}}{J^{\prime \prime 2}}\right)|A+\beta|^{2}\right]\right\}=0,
\end{aligned}
$$

can be satisfied when $J^{\prime}=0$, which separates the Polonyi multiplet from the vector multiplet. The Polonyi field VEV is then given by $\langle A\rangle=(\sqrt{3}-1)$ and $\beta=2-\sqrt{3}$ [9]. This solution describes a stable Minkowski vacuum with spontaneous SUSY breaking at an arbitrary scale $\langle F\rangle=\mu$. The related gravitino mass (at the minimum having $J^{\prime}=0$ ) is given by $m_{3 / 2}=\mu e^{2-\sqrt{3}}$. There is also a massive scalar of mass $2 m_{3 / 2}$ and a massless fermion in the physical spectrum.

As a result, the Polonyi field does not affect the inflation driven by the inflaton scalar $C$ belonging to the massive vector multiplet and having the $D$-type scalar potential $V(C)=\frac{1}{2} J^{\prime 2}$ with a real $J$-function. Of course, the true inflaton field should be canonically normalized via the proper field redefinition of $C$.

\section{Massless vector multiplet and super-Higgs mechanism}

The matter-coupled supergravity model (1) can also be considered as a supersymmetric (Abelian, non-minimal) gauge theory (coupled to supergravity and a Higgs superfield) in the (supersymmetric) gauge where the Higgs superfield is gauged away (say, equal to 1). When the gauge $U(1)$ symmetry is restored by introducing back the Higgs (chiral) superfield, the vector superfield $V$ becomes the gauge superfield of a spontaneously broken $U$ (1) gauge group. In this Section we restore the gauge symmetry in the way consistent with local supersymmetry, and then compare our results with those of the previous Section.

We start with a Lagrangian having the same form as (1),

$$
\begin{aligned}
\mathcal{L}= & \int \mathrm{d}^{2} \theta 2 \mathcal{E}\left\{\frac{3}{8}(\overline{\mathcal{D D}}-8 \mathcal{R}) e^{-\frac{1}{3}(K+2 J)}+\frac{1}{4} W^{\alpha} W_{\alpha}+\mathcal{W}\left(\Phi_{i}\right)\right\} \\
& + \text { h.c. },
\end{aligned}
$$

where $K=K\left(\Phi_{i}, \bar{\Phi}_{j}\right)$ and the indices $i, j, k$ refer to the chiral (matter) superfields, excluding the Higgs chiral superfield, which we denote $H, \bar{H}$. Now, in contrast to the previous section, the real function $J$ also depends on the Higgs superfield as $J=J\left(H e^{2 V} \bar{H}\right)$, while the vector superfield $V$ is massless. The Lagrangian (9) is invariant under the supersymmetric $U(1)$ gauge transformations

$$
\begin{aligned}
H \rightarrow H^{\prime} & =e^{-i Z} H, \quad \bar{H} \rightarrow \bar{H}^{\prime}=e^{i \bar{Z}} \bar{H}, \\
V \rightarrow V^{\prime} & =V+\frac{i}{2}(Z-\bar{Z}),
\end{aligned}
$$

whose gauge parameter $Z$ itself is a chiral superfield. The Lagrangian (1) of Sect. 2 is recovered from Eq. (9) in the gauge $H=1$, after the redefinition $J_{\text {new }}\left(e^{2 V}\right)=J_{\text {old }}(V)$.
The $U(1)$ gauge symmetry of the Lagrangian (9) allows us to choose a different (Wess-Zumino) supersymmetric gauge by "gauging away" the chiral and anti-chiral parts of the general superfield $V$ via the appropriate choice of the superfield parameters $Z$ and $\bar{Z}$,

$V\left|=\mathcal{D}_{\alpha} \mathcal{D}_{\beta} V\right|=\overline{\mathcal{D}}_{\dot{\alpha}} \overline{\mathcal{D}}_{\dot{\beta}} V \mid=0$,

$\overline{\mathcal{D}}_{\dot{\alpha}} \mathcal{D}_{\alpha} V \mid=\sigma_{\alpha \dot{\alpha}}{ }^{m} B_{m}$,

$\mathcal{D}_{\alpha} W^{\beta} \mid=\frac{1}{4} \sigma_{\alpha \dot{\alpha}}{ }^{m} \bar{\sigma}^{\dot{\alpha} \beta n}\left(2 i F_{m n}\right)+\delta_{\alpha}{ }^{\beta} D$,

$\overline{\mathcal{D D}} \mathcal{D} \mathcal{D} V \mid=\frac{16}{3} b^{m} B_{m}+8 D$,

where the vertical bars denote the leading field components of the superfields.

It is straightforward (but tedious) to calculate the bosonic part of the Lagrangian in terms of the superfield components in an Einstein frame, after elimination of the auxiliary fields and Weyl rescaling. We find

$$
\begin{aligned}
e^{-1} \mathcal{L}= & -\frac{1}{2} R-K_{i j^{*}} \partial^{m} A_{i} \partial_{m} \bar{A}_{j}-\frac{1}{4} F_{m n} F^{m n} \\
& -2 J_{h \bar{h}} \partial_{m} h \partial^{m} \bar{h}-\frac{1}{2} J_{V^{2}} B_{m} B^{m} \\
& +i B_{m}\left(J_{V h} \partial^{m} h-J_{V \bar{h}} \partial^{m} \bar{h}\right)-\mathcal{V}
\end{aligned}
$$

where $h, \bar{h}$ are the Higgs field and its conjugate. We use the notation $J_{h \bar{h}} \equiv \frac{\partial^{2} J}{\partial h \partial \bar{h}}\left|, J_{V h} \equiv \frac{\partial^{2} J}{\partial h \partial V}\right|$ and $J_{V^{2}} \equiv \frac{\partial^{2} J}{\partial V^{2}} \mid$. As regards the scalar potential, we get

$$
\begin{aligned}
\mathcal{V}= & \frac{1}{2} J_{V}^{2}+e^{K+2 J}\left\{(K+2 J)^{I J^{*}}\left(W_{I}+(K+2 J)_{I} W\right)\right. \\
& \left.\times\left(\bar{W}_{J^{*}}+(K+2 J)_{J^{*}} \bar{W}\right)-3 W \bar{W}\right\}
\end{aligned}
$$

where the capital Latin indices $I, J$ collectively denote all chiral superfields (as well as their lowest field components) including the Higgs superfield.

The standard $U(1)$ Higgs mechanism setting appears after employing the canonical function $J=\frac{1}{2} h e^{2 V} \bar{h}$. As regards the Higgs sector, it leads to

$$
\begin{aligned}
e^{-1} \mathcal{L}_{\text {Higgs }}= & -\partial_{m} h \partial^{m} \bar{h}+i B_{m}\left(\bar{h} \partial^{m} h-h \partial^{m} \bar{h}\right) \\
& -h \bar{h} B_{m} B^{m}-\mathcal{V} .
\end{aligned}
$$

When parameterizing $h$ and $\bar{h}$ as

$h=\frac{1}{\sqrt{2}}(\rho+v) e^{i \zeta}, \quad \bar{h}=\frac{1}{\sqrt{2}}(\rho+v) e^{-i \zeta}$,

where $\rho$ is the (real) Higgs boson, $v \equiv\langle h\rangle=\langle\bar{h}\rangle$ is the Higgs $\mathrm{VEV}$, and $\zeta$ is the Goldstone boson, in the unitary gauge of $h \rightarrow h^{\prime}=e^{-i \zeta} h$ and $B_{m} \rightarrow B_{m}^{\prime}=B_{m}+\partial_{m} \zeta$, we reproduce the standard result [12], 
$e^{-1} \mathcal{L}_{\text {Higgs }}=-\frac{1}{2} \partial_{m} \rho \partial^{m} \rho-\frac{1}{2}(\rho+v)^{2} B_{m} B^{m}-\mathcal{V}$.

The same result is also achieved by considering the superHiggs mechanism where, in order to get rid of the Goldstone mode, we employ the super-gauge transformations (10) and (11), and define the relevant field components of $Z$ and $i(Z-$ $\bar{Z})$ as

$Z\left|=\zeta+i \xi, \quad{ }^{i} \overline{\mathcal{D}}_{\dot{\alpha}} \mathcal{D}_{\alpha}(Z-\bar{Z})\right|=\sigma_{\alpha \dot{\alpha}}^{m} \partial_{m} \zeta$

Examining the lowest components of the transformation (10), we find that the real part of $Z \mid$ and $\bar{Z} \mid$ cancels the Goldstone mode of (15). Similarly, applying the derivatives $\overline{\mathcal{D}}_{\dot{\alpha}}$ and $\mathcal{D}_{\alpha}$ to (11) and taking their lowest components (recalling then $\overline{\mathcal{D}}_{\dot{\alpha}} \mathcal{D}_{\alpha} V \mid=\sigma_{\alpha \dot{\alpha}}^{m} B_{m}$ ), we conclude that the vector field "eats up" the Goldstone mode indeed, as

$B_{m}^{\prime}=B_{m}+\partial_{m} \zeta$

\section{Adding a cosmological constant}

A cosmological constant (or dark energy) can be introduced into our framework without breaking any symmetries, via a simple modification of the Polonyi sector and its parameters $\alpha$ and $\beta$ introduced in Sect. 2. ${ }^{3}$

Just adding a (very) small positive constant $\delta$ and assuming that $J^{\prime}=0$ at the minimum of the potential modify the (Minkowski) vacuum condition $V=0$ of Sect. 2 to

$V=\mu^{2} e^{\alpha^{2}} \delta=m_{3 / 2}^{2} \delta$.

By comparing the condition (19) to Eq. (6) we find the relation

$\left(1+\alpha \beta+\alpha^{2}\right)^{2}-3(\alpha+\beta)^{2}=\delta$.

A solution to Eqs. (20) and (7) with $V=m_{3 / 2}^{2} \delta$ is the true minimum, and it reads

$$
\begin{aligned}
& \alpha=(\sqrt{3}-1)+\frac{3-2 \sqrt{3}}{3(\sqrt{3}-1)} \delta+\mathcal{O}\left(\delta^{2}\right), \\
& \beta=(2-\sqrt{3})+\frac{\sqrt{3}-3}{6(\sqrt{3}-1)} \delta+\mathcal{O}\left(\delta^{2}\right) .
\end{aligned}
$$

This yields a de Sitter vacuum with the spontaneously broken SUSY after inflation.

Inserting the solution into the superpotential and ignoring the $\mathcal{O}\left(\delta^{2}\right)$-terms, we find

\footnotetext{
3 A similar idea was used in Ref. [13], though in the different context, where the Polonyi potential was needed to prevent the real part of the stabilizer field from vanishing at the minimum by imposing the condition $m_{\text {gravitino }} \ll m_{\text {inflaton }}$. In our approach, there is no stabilizer field, while the inflation comes from the D-type potential.
}

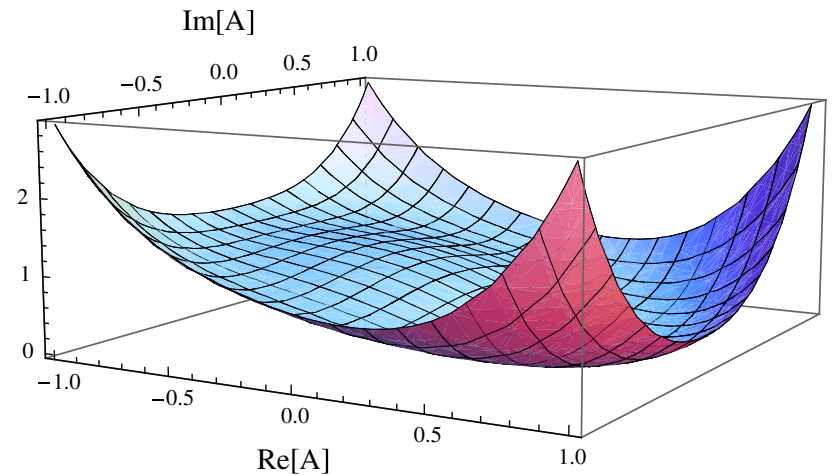

Fig. 1 The scalar potential $\tilde{V}=\mu^{-2} e^{-A \bar{A}-2 J} V$ as a function of $\operatorname{Re}(A)$ and $\operatorname{Im}(A)$ at $J^{\prime}=0$

$\langle\mathcal{W}\rangle=\mu(\alpha+\beta)=\mu\left(a+b-\frac{1}{2} \delta\right)$,

where $a \equiv(\sqrt{3}-1)$ and $b \equiv(2-\sqrt{3})$ are the SUSY breaking vacuum solutions to the Polonyi parameters in the absence of a cosmological constant (Sect. 2).

\section{Scalar potential and vacuum stability}

For completeness, the stability of our vacuum solutions should also be examined. On the one hand, in our model the vacuum stability is almost guaranteed because both functions $J^{\prime 2}$ and $J^{\prime \prime}$ enter the scalar potential

$$
\begin{aligned}
V= & \frac{1}{2} J^{\prime 2}+\mu^{2} e^{A \bar{A}+2 J} \\
& \times\left[|1+A \beta+A \bar{A}|^{2}-\left(3-2 \frac{J^{\prime 2}}{J^{\prime \prime}}\right)|A+\beta|^{2}\right]
\end{aligned}
$$

with the positive sign, while the function $J^{\prime \prime}$ is required to be positive for the ghost-freedom. On the other hand, the only term with the negative sign in the scalar potential (23) is $-3|A+\beta|^{2}$ but it grows slower than the positive quartic term $|1+A \beta+A \bar{A}|^{4}$.

The non-negativity of the scalar potential (23) for $|A|<1$ is not as apparent as that for $|A| \geq 1$. That is why we supply Figs. 1 and 2 where the non-negativity becomes apparent too. In accordance to the previous Sect. 4, we can also add a positive cosmological constant that shifts the minimum to $V=m_{3 / 2}^{2} \delta$ describing a de Sitter vacuum.

\section{Conclusion}

Our new results are given in Sects. 3, 4 and 5. The new gaugeinvariant formulation of our models can be used for unification of inflation with super-GUT in the context of supergravity, and it has a single inflaton scalar field having a positive 


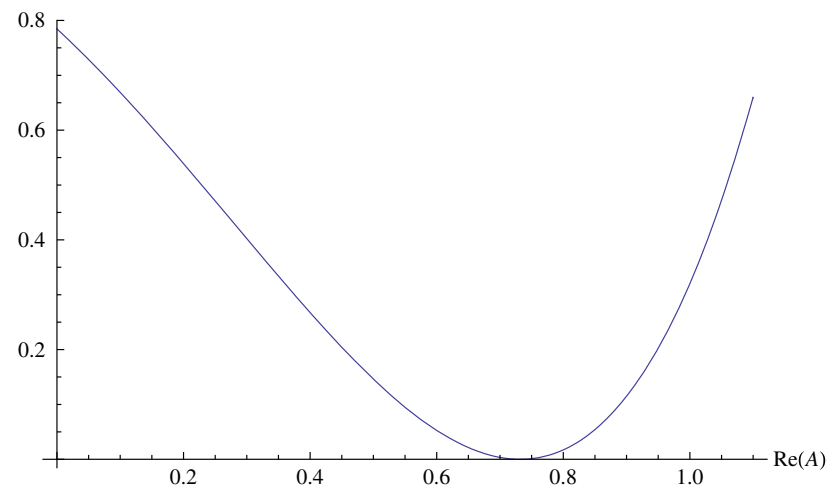

Fig. 2 The real slice at $\operatorname{Im}(A)=0$ of Fig. 1 around the minimum of $\tilde{V}$

definite scalar potential, a spontaneous SUSY breaking and a de Sitter vacuum after inflation. Our approach does not preserve the $R$-symmetry.

Our upgrade of the earlier results in Ref. [8] is not limited to the generalized matter couplings in supergravity, given by Eqs. (12) and (13). The standard approach to inflation in supergravity is based on the assumption that the inflaton belongs to a chiral (scalar) multiplet. It leads to the well-known problems such as the so-called $\eta$-problem, stabilization of other scalars, getting SUSY breaking and a dS vacuum after inflation, etc. Though some solutions to these problems exist in the literature, they are rather complicated and include the additional "hand-made" input such as extra (stabilizing) matter superfields, extra (shift) symmetries or extra (nilpotency) conditions. We advocate another approach where inflaton is assumed to belong to a massive vector multiplet, while SUSY breaking and a dS vacuum are achieved with the help of a Polonyi superfield. It is much simpler and more flexible than the standard approach.

Physical applications of our approach to super-GUT and reheating are crucially dependent upon the way how the fields present in our models interact with the super-GUT fields. Consistency of sGUT with inflation may lead to some new constraints on both. For instance, inflaton couplings to other matter have to be smaller than $10^{-3}$, in order to preserve flatness of the inflaton scalar potential and match the observed spectrum of CMB density perturbations. In particular, Yukawa couplings of inflaton to right-handed (sterile) neutrino are crucial in addressing leptogenesis via inflaton decay and the subsequent reheating via decays of the righthanded neutrino into visible particles of the Standard Model. Unfortunately, all this appears to be highly model-dependent at present. A derivation of our supergravity models from superstrings, if any, is desirable because it would simultaneously fix those (unknown) interactions and thus provide specific tools for a computation of reheating temperature, matter abundance, etc. after inflation, together with the low-energy predictions via gravity- or gauge-mediated SUSY breaking to the electro-weak scale - see e.g., Ref. [14] for the previous studies along these lines.

Our models can be further extended in the gauge sector to the Born-Infeld-type gauge theory coupled to supergravity and other matter, along the lines of Refs. $[15,16]$, thus providing further support toward their possible origin in superstring (flux-) compactification.

Acknowledgements Y.A. is supported by a scholarship from the Ministry of Education, Culture, Sports, Science and Technology (MEXT) in Japan. S.V.K. is supported by a Grant-in-Aid of the Japanese Society for Promotion of Science (JSPS) under No. 26400252, a TMU President Grant of Tokyo Metropolitan University in Japan, the World Premier International Research Center Initiative (WPI Initiative), MEXT, Japan, and the Competitiveness Enhancement Program of Tomsk Polytechnic University in Russia. The authors are grateful to the referees for their critical comments.

Open Access This article is distributed under the terms of the Creative Commons Attribution 4.0 International License (http://creativecomm ons.org/licenses/by/4.0/), which permits unrestricted use, distribution, and reproduction in any medium, provided you give appropriate credit to the original author(s) and the source, provide a link to the Creative Commons license, and indicate if changes were made.

Funded by $\mathrm{SCOAP}^{3}$.

\section{References}

1. Planck Collaboration, P.A.R. Ade et al., Planck 2015 results. XIII. Cosmological parameters. arXiv:1502.01589 [astro-ph.CO]

2. Planck Collaboration, P.A.R. Ade et al., Planck 2015 results. XX. Constraints on inflation. arXiv:1502.02114 [astro-ph.CO]

3. BICEP2, Keck Array Collaboration, P.A.R. Ade et al., Improved constraints on cosmology and foregrounds from BICEP2 and Keck array cosmic microwave background data with inclusion of 95 GHz band. Phys. Rev. Lett. 116, 031302 (2016). arXiv: 1510.09217 [astro-ph.CO]

4. M. Yamaguchi, Supergravity based inflation models: a review. Class. Quant. Grav. 28, 103001 (2011). arXiv:1101.2488 [astroph.CO]

5. S.V. Ketov, Supergravity and early universe: the meeting point of cosmology and high-energy physics. Int. J. Mod. Phys. A 28, 1330021 (2013). arXiv:1201.2239 [hep-th]

6. S. Ferrara, R. Kallosh, A. Linde, M. Porrati, Minimal supergravity models of inflation. Phys. Rev. D 88(8), 085038 (2013). arXiv: 1307.7696 [hep-th]

7. A. Van Proeyen, Massive vector multiplets in supergravity. Nucl. Phys. B 162, 376 (1980)

8. Y. Aldabergenov, S.V. Ketov, SUSY breaking after inflation in supergravity with inflaton in a massive vector supermultiplet. Phys. Lett. B 761, 115 (2016). arXiv:1607.05366 [hep-th]

9. J. Polonyi, Generalization of the Massive Scalar Multiplet Coupling to the Supergravity. Hungary Cent. Inst. Res. KFKI-77-93 (1977, REC. JUL 1978), 5 p. KFKI-77-93, unpublished

10. S.V. Ketov, Solitons, monopoles and duality: from SineGordon to Seiberg-Witten. Fortsch. Phys. 45, 237 (1997). arXiv:hep-th/9611209

11. J. Wess, J. Bagger, Supersymmetry and Supergravity (Princeton University Press, Princeton, NJ, 1992)

12. S. Weinberg, General theory of broken local symmetries. Phys. Rev. D 7, 1068 (1973) 
13. A. Linde, On inflation, cosmological constant, and SUSY breaking. JCAP 1611, 002 (2016). arXiv:1608.00119 [hep-th]

14. J. Ellis, H.-J. He, Z.-Z. Xianyu, Higgs inflation, reheating and gravitino production in no-scale supersymmetric GUTs. JCAP 1808, 068 (2016). arXiv:1606.02202 [hep-th]
15. H. Abe, Y. Sakamura, Y. Yamada, Massive vector multiplet inflation with Dirac-Born-Infeld type action. Phys. Rev. D 91, 125042 (2015). arXiv:1505.02235 [hep-th]

16. S. Aoki, Y. Yamada, More on DBI action in $4 \mathrm{D} \mathcal{N}=1$ supergravity. arXiv:1611.08426 [hep-th] 